\title{
Probing the nature of possible open cluster remnants with the Southern Proper Motion Program
}

\author{
G. Carraro ${ }^{1,2,3}$, D. I. Dinescu ${ }^{2}$, T. M. Girard ${ }^{2}$, and W. F. van Altena ${ }^{2}$ \\ 1 Departamento de Astronomía, Universidad de Chile, Casilla 36-D, Santiago, Chile \\ e-mail: gcarraro@das.uchile.cl \\ 2 Astronomy Department, Yale University, PO Box 208101, New Haven, CT 06520-8101, USA \\ 3 Dipartimento di Astronomia, Università di Padova, Vicolo Osservatorio 2, 35122 Padova, Italy
}

Received 21 September 2004 / Accepted 29 November 2004

\begin{abstract}
We discuss the nature of eleven Possible Open Cluster Remnants (POCRs) by using absolute proper motions from the Southern Proper Motion (SPM) Program 3 (Girard et al. 2004, AJ, 127, 3060) combined with near infrared photometry from 2MASS. The analysis is done by considering the distribution of stars in the Color-Magnitude and the Vector Point diagrams. We successfully probed the capabilities of the SPM catalog to detect a physical group by looking at the open cluster Blanco 1. However, within the uncertainties of the SPM3 catalog and basing on 2MASS photometry we conclude that only one - ESO 282SC26 - out of eleven objects turns out to be a probable physical group. We suggest it is an open cluster 1.3 Gyr old and located $1.4 \mathrm{kpc}$ from the Sun.
\end{abstract}

Key words. open clusters and associations : general - astrometry

\section{Introduction}

The recently published third release of the Southern Proper Motion (SPM3) Program contains an almost complete cata$\log$ of absolute proper motions of stars located in the southern hemisphere between declination of $-20^{\circ}$ and $-45^{\circ}$ down to $V=17.5$ (Girard et al. 2004) ${ }^{1}$. The typical uncertainty in the proper motion components is 4.0 mas $\mathrm{yr}^{-1}$. The proper motions are on the International Celestial Reference System by way of Hipparcos Catalog stars, and have an estimated systematic un-

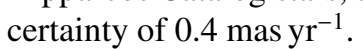

This catalog therefore represents a valuable tool to search for newly, unknown open clusters like in the case of the Hipparcos catalog (Platais et al. 1998) and to investigate the nature of objects like the so-called Possible Open Cluster Remnants (POCRS), proposed by Bica et al. (2001) to be candidate open clusters in advanced stages of dynamical evolution, just before their dissolution and merging with the general Galactic disk field. These objects were selected through star counts, and are located rather high onto the Galactic plane (latitude larger than $15^{\circ}$ ), where one does not expect to find any particular star over-density. Bica et al. (2001) provide coordinates and diameters for a list of 34 clusters, some of which have NGC identification.

Some of them have already been studied in details. NGC 6994 (M73) was proved to be just a random enhancements of four bright stars by Carraro (2000) and

\footnotetext{
${ }^{1}$ http://www.astro.yale.edu/astrom/spm3cat/spm3.html
}

Odenchirken \& Soubiran (2002). Recently, Villanova et al. (2003, 2004a) demonstrate that NGC 5385, NGC 2664 and Collinder 21 are as well random alignments of field stars. On the contrary, NGC 1901 was proved to be a genuine star cluster by Pavani et al. (2001) and Villanova et al. (2004b). In all cases the nature of the objects was clarified by carefully looking at the distribution of proper motions and radial velocities, and therefore at the kinematics of the candidate member stars.

Since in most cases these objects are identified by clumps of bright stars, we searched the SPM3 catalog and actually found proper motions for a significant number of stars (in the range 100-2000) in 11 POCRs extracted from Bica et al. (2001) list (see Table 1 for details). The SPM3 catalog together with absolute proper motions contains also photographic $B$ and $V$ magnitudes, which unfortunately are affected by large errors, and therefore are not very useful to build up good Color Magnitude Diagrams (CMDs). However, the SPM3 catalog has been combined with the 2MASS (Skrutskie et al. 1997) one, thus providing for nearly all the objects near-infrared $J, H$ and $K$ photometry.

An analysis similar to the one presented here was done by Baumgardt (1998), who was able to clarify the nature of 8 controversial open clusters by analyzing their parallaxes, proper motion components and $B$ and $V$ magnitudes from the Hipparcos catalog, and by Baumgardt et al. (2000) and Dias et al. (2001, 2002a).

The analysis of the clusters is performed by comparing the proper motion distribution and the CMDs of the stars in the 
Table 1. Basic parameters of the objects under investigation. Coordinates are for J2000.0 equinox and have been taken from Dias et al. (2002b).

\begin{tabular}{cccccc}
\hline \hline Name & RA & Dec & $l$ & $b$ & Diam \\
\hline & hh $: \mathrm{mm}: \mathrm{ss}$ & ${ }^{\circ}:^{\prime}:^{\prime \prime}$ & {$[\mathrm{deg}]$} & {$[\mathrm{deg}]$} & arcmin \\
\hline ESO 486SC45 & $05: 16: 43$ & $-24: 02: 17$ & 226.07 & -30.80 & 3.5 \\
NGC 1891 & $05: 21: 25$ & $-35: 44: 24$ & 239.69 & -32.87 & 15.0 \\
NGC 1963 & $05: 32: 17$ & $-36: 23: 30$ & 240.99 & -30.86 & 13.0 \\
ESO 424SC25 & $05: 49: 49$ & $-32: 28: 20$ & 237.72 & -26.37 & 9.0 \\
ESO 425SC06 & $06: 04: 50$ & $-29: 10: 59$ & 235.39 & -22.28 & 6.0 \\
ESO 425SC15 & $06: 14: 35$ & $-29: 22: 30$ & 236.37 & -20.35 & 6.0 \\
ESO 437SC61 & $10: 48: 03$ & $-29: 23: 26$ & 273.06 & 26.22 & 5.01 \\
ESO 442SC04 & $12: 34: 05$ & $-29: 24: 38$ & 298.40 & 33.30 & 11.0 \\
IC 1023 & $14: 32: 25$ & $-35: 48: 13$ & 324.95 & 22.71 & 5.0 \\
ESO 282SC26 & $19: 13: 52$ & $-42: 38: 58$ & 335.01 & -21.89 & 15.0 \\
ESO 464SC09 & $20: 59: 37$ & $-29: 23: 12$ & 15.92 & -39.43 & 4.0 \\
\hline Blanco 1 & $00: 04: 07$ & $-29: 50: 00$ & 15.57 & -79.26 & 90 \\
\hline
\end{tabular}

POCR area and in an off-set field (typically half a degree apart). The main aim of this study is to clarify the nature of these POCRs, and in case we found that one object is a probable physical cluster, we would provide for it the first estimate of its fundamental parameters, and a list of candidate members for spectroscopic follow-up.

The layout of this paper is as follows.

In Sect. 2 we test SPM3 capabilities against the open cluster Blanco 1. In Sect. 3 we describe the analysis method, and in Sect. 4 we discuss individually all the candidates. Our conclusions are finally highlighted in Sect. 5.

\section{Testing SPM3 capabilities: The open cluster Blanco 1}

All the fields here considered are located high onto the galactic plane, and therefore several galaxies are easily encountered. We removed all the extended sources from the catalog before commencing the analysis.

To probe the capabilities of SPM3 to detect a physical group, we searched for a well known, genuine open cluster in the catalog, and we found the open cluster Blanco 1. This is a young nearby cluster, $290 \mathrm{pc}$ from the Sun, with a diameter of $2.5 \mathrm{deg}$, and with mean proper motions $\mu_{\alpha}=20.06 \pm 0.49$ and $\mu_{\delta}=3.44 \pm 0.25$ mas yr $^{-1}$ (Van Leeuwen 1999; Dias et al. 2001).

We extracted from the SPM3 catalog 6225 stars in an area of 2.5 squared deg. centered on Blanco 1 nominal center (see Table 1), and 4677 stars in a similar size area 5 degrees northward of the cluster. The results are shown in Fig. 1, where we present the trend of proper motion components versus $K$ mag. The left panels show the distribution of Blanco 1 stars, while the right panels show the distribution of stars in the off-set field. The solid lines in the left panels are the Hipparcos mean proper motion component (Van Leeuwen 1999). First of all we note that the proper motion components start to be significantly scattered at $K \approx 11.0$. However, we clearly see a significant stars concentration at $9.0 \leq K \leq 12.0$ in both the proper motion components, close to the expected mean motion component of the cluster from Hipparcos (solid lines), although a sizeable

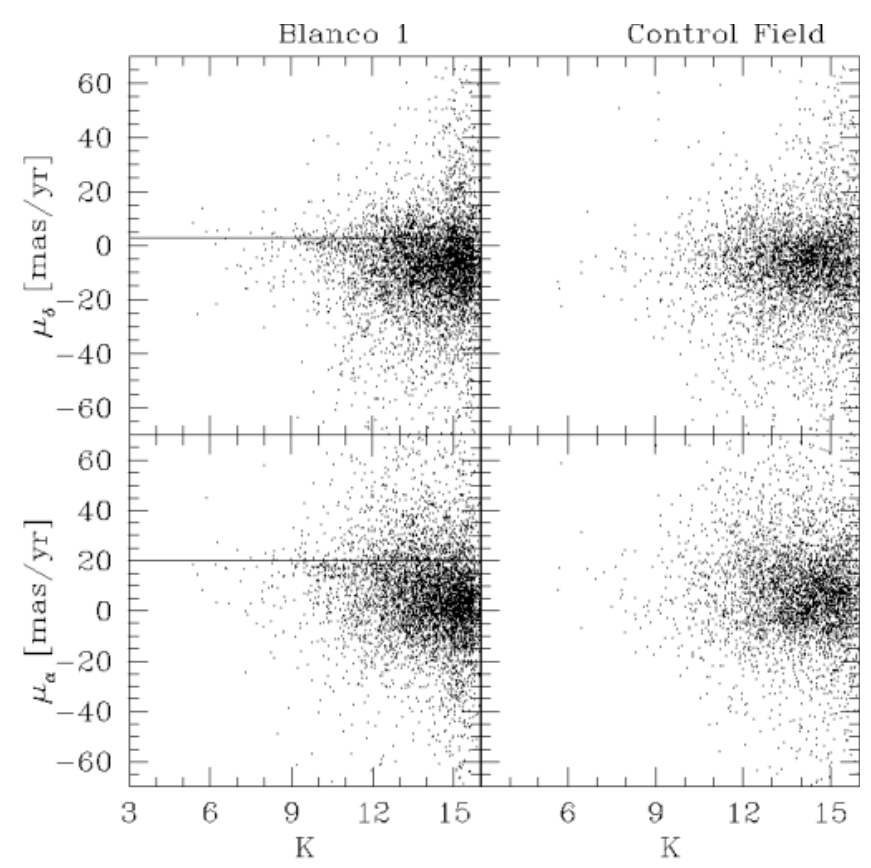

Fig. 1. Trend of proper motion components versus $K$ mag. in Blanco 1 (left panels) and a nearby field (right panels). The solid line is Blanco 1 mean proper motion components taken from Dias et al. (2001).

difference is visible in the case of $\mu_{\alpha}$. These stars probably constitute the cluster Blanco 1.

Since the precision $(1 \sigma)$ of the SPM3 catalog is about 4 mas $^{-1} r^{-1}$, we picked up from the cluster plots all the stars which lie within a strip 16 mas yr$^{-1}$ wide $(2 \times \sigma)$ centered at Blanco 1 proper motion mean values, where we expect to find $95 \%$ of member stars within our uncertainties. The result is shown in Fig. 2, where we plot the 2MASS CMD of Blanco 1. In the left panel we plot all the stars detected in the cluster area, whereas in the right panel we plot all the stars found in the control field. The stars selected on a proper motion basis are plotted in the left panel as filled circles, and they clearly define a nice Main Sequence (MS) with some scatter. The dashed line in this plot is to guide the eye, and it is a Zero Age Main 


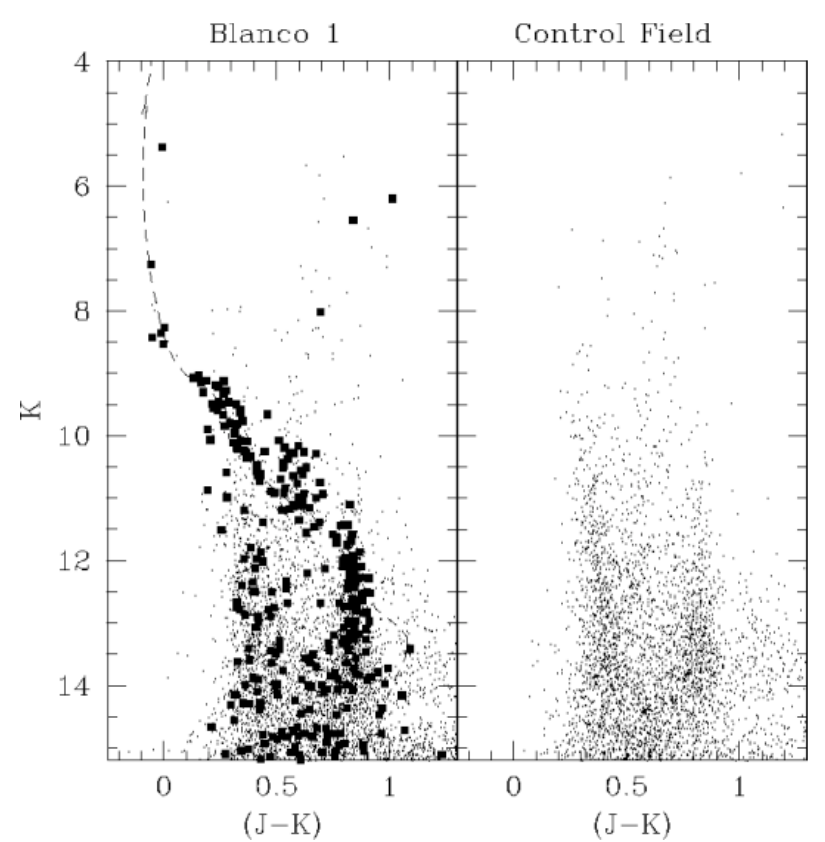

Fig. 2. Left panel: CMD of Blanco 1 from 2MASS. Probable members are shown as filled circles. The dashed line is the Schmidt-Kaler (1982) ZAMS shifted by the cluster distance modulus and reddening. Right panel: CMD of a nearby field from 2MASS.

Sequence (ZAMS) from Schmidt-Kaler (1982) for the distance $(m-M=7.1)$ and the reddening $(E(J-K)=0.019)$ of Blanco 1 .

Finally, in Fig. 3 we show the VPD for the stars brighter than $K=13.5$ in the Blanco 1 field (left panel) and in the offset field (right panel). The cut off in magnitude is mainly motivated by the need to limit the field star contamination; however it is the population brighter than this cut-off that actually better defines the cluster in Fig. 2. In the VPD the cluster readily appears as a concentration of stars centered at $\mu_{\alpha} \approx 20$. and $\mu_{\delta} \approx 3$., and this clustering does not have a counterpart in the off-set field. If we imagine to remove the cluster, the stars distribution in the VPD is the same both for the cluster and for the off-set field.

However, since in general OCRs are less populated ensembles of stars and occupy smaller areas than Blanco 1, we decide to further check SPM 3 capabilities on a smaller area in the open cluster Blanco 1, with the aim to mimick the case of an OCR. We searched the SPM 3 catalogue in a $15^{\prime} \times 15^{\prime}$ area centered in Blanco 1, and we extracted 101 stars in the cluster area, and 108 stars from a same area off-set field.

The results are shown in Figs. 4 and 5, where we plot the VPD and the CMD, respectively. Even considering a smaller area and a significanly smaller number of stars, the open cluster Blanco 1 is readily detected as a clump of stars around the mean proper motions in the VPD (Fig. 4), and as a poorly sampled MS in the CMD (Fig. 5). We derived the mean proper motion for the probable member stars (14, in this smaller field), and they turned out to be $\mu_{\alpha}=19.63 \pm 3.44$ ( 1 standard deviation) and $\mu_{\delta}=4.21 \pm 4.14$ ( 1 standard deviation). These values are in good agreement with Van Leeuwen (1999) ones, although the dispersion is larger, but well consistent with the SPM 3 uncertainties.

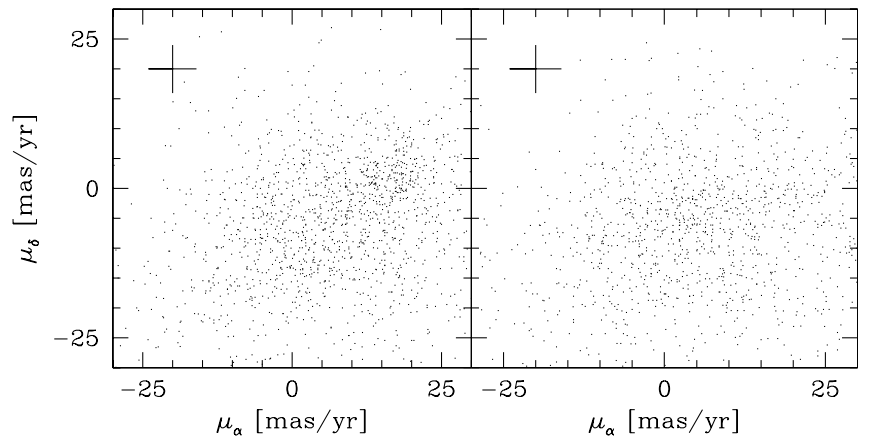

Fig. 3. Left panel: VPD from SPM3 for all the stars brighter than $K=13.5$ in the field of Blanco 1. Right panel: the same as left panel, but for the nearby field. The cross indicates the $1 \sigma$ error bar from SPM3.

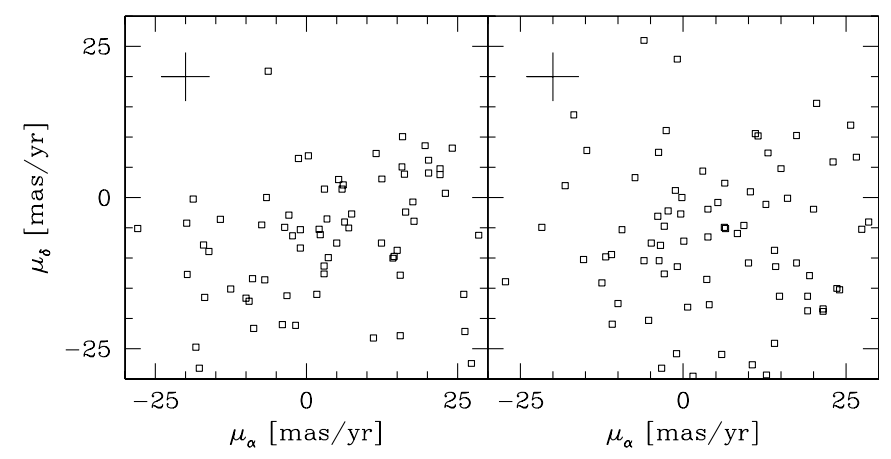

Fig. 4. Left panel: VPD from SPM3 for all the stars brighter than $K=13.5$ in the smaller field of Blanco 1. Right panel: the same as left panel, but for the smaller area nearby field. The cross indicates the $1 \sigma$ error bar from SPM3.

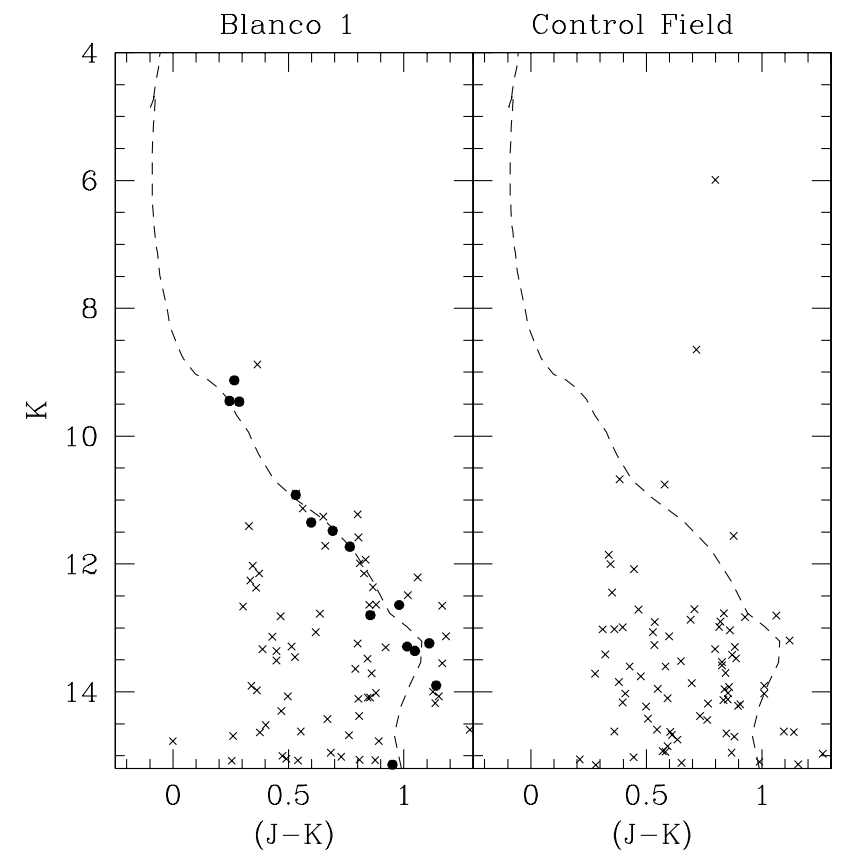

Fig. 5. Left panel: CMD of Blanco 1 from 2MASS in an area of $15^{\prime} \times 15^{\prime}$. Probable members are shown as filled circles. The dashed line is the Schmidt-Kaler (1982) ZAMS shifted by the cluster distance modulus and reddening. Right panel: CMD of a nearby same area field from 2MASS. 
All these plots demonstrate that the absolute proper motions from SPM3 combined with 2MASS photometry are capable to identify a physical group of stars with the same tangential motions.

Therefore it provides us with a tool to be used when looking for suggested physical groups like POCRs.

\section{Analysis of POCRs: The method}

All the targets of the present study are over-densities of bright stars with respect to the surrounding field (Bica et al. 2001). This is a necessary condition for a bound group of stars to exist.

However, only if these stars share common motion and the common motion stars exhibit distinctive features in the CMD, we are going to conclude that the stars actually form a physical system (Platais et al. 1998).

On the contrary, if neither they show common motion nor known features in the CMD, we must conclude that these stars are simple random star accumulations along the line of sight.

In the following we are going to apply the same technique as for Blanco 1 to the POCRs listed in Table 1. In details, we compare the target field with a control field taken in all the cases at a distance of half a degree from the nominal target center.

At odds with Blanco 1, here we do not know the object proper motions, and therefore we look for possible member

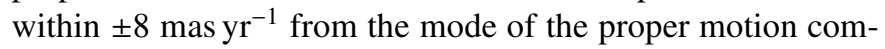
ponents distribution, both in the target and in the control field. This way, we hope to catch most of the object members, if any. The searching area depends on the cluster radius, as listed in Table 1, but in all cases is much larger than the cluster diameters as proposed by Bica et al. (2001).

In the following we are showing how each POCR candidate and the corresponding off-set field look like in the VPD and CMD. This is to show the reader how we basically decided upon the probable presence of a cluster. Basically we search for possible differences in the proper motions between target and field, and look for distinctive features in the CMD of common motions stars.

We searched in the SPM3 catalogue for stars brighter than $K=14$, and considered only the stars with proper motions

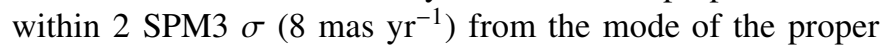
motion components distribution computed taking into account all the detected stars.

The adopted limiting magnitude allow us to detect the brightest candidate stars and limit the field star contamination, which increases at increasing magnitude. Down to $K=14$ the 2MASS photometry is very reliable, and we do not expect to find many OCR members at fainter magnitudes, since the samll mass stars quickly escape the cluster. Besides, the magnitude range of OCR candidate stars is very variable, some of them having very bright stars, and other having fainter stars which produce the overdensity above the Galactic field in a way that one can hope also to find a few more faint members.
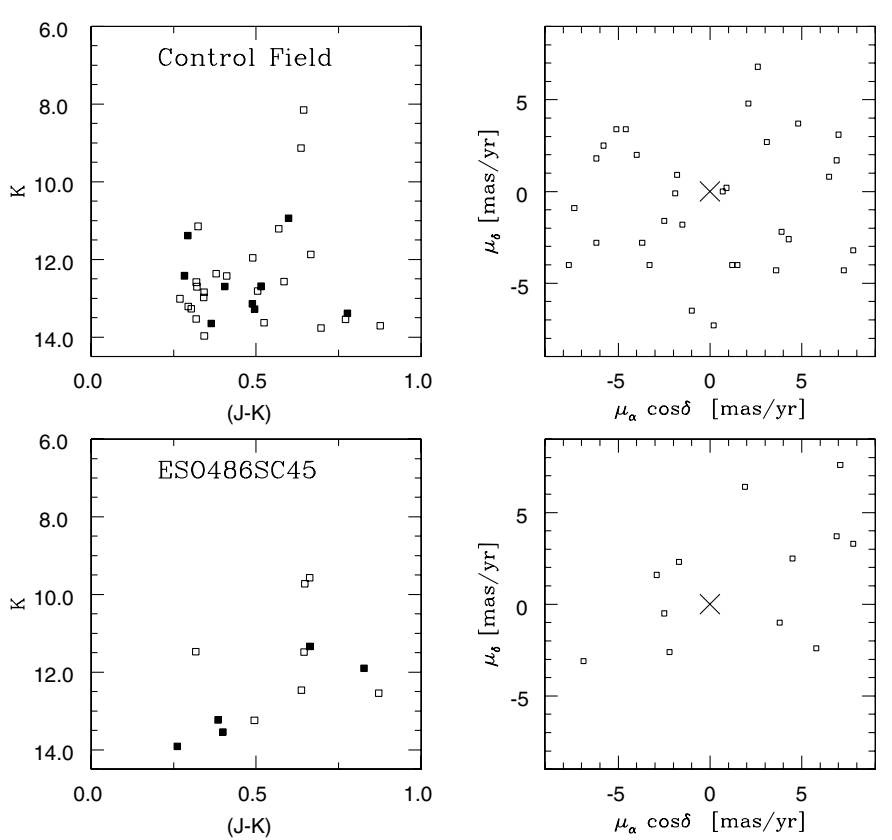

Fig. 6. The ESO 486SC45 group. Only stars within 2 SPM $3 \sigma$ from the proper motion distributions mode are plotted. Bottom left panels: 2MASS CMD. Filled symbols indicate stars with proper motions within $1 \sigma$ from the proper motion distributions mode, open symbols stars with proper motion within $2 \sigma$ from the proper motion distributions mode. Bottom right panel: VPD; the cross is centered in the field proper motions mode. Upper left panel: same as the bottom left panel, but for the control field; Upper right panel: same as the bottom right panel, but for the control field.

\section{Individual objects analysis}

In this section we provide for each object some details of the searching procedure and the corresponding VPDs and CMDs for the target and control fields.

\subsection{ESO $486 S C 45$}

This object is shown in Fig. 6 . We considered a $15^{\prime} \times 15^{\prime}$ area, and we found 142 stars in the object field, and 154 stars in the control field. The mode of the proper motion distribution turned out to be around $\mu_{\alpha}=0.0$ and $\mu_{\delta}=0.0 \mathrm{mas} \mathrm{yr}^{-1}$ both in the target and in the control field, and the control field looks less scattered than the cluster. By inspecting Fig. 6, we do not see any of the features which would lead us to think about the presence of a cluster, namely neither a concentration in the VPDs which look very scattered, nor a sequence in the CMDs. In this case the field seems to have more common motion stars than the target.

We therefore conclude that ESO 486SC45 is not a physical group.

\subsection{NGC 1891}

This target is presented in Fig. 7. We considered a $30^{\prime} \times 30^{\prime}$ area, and we found 379 stars in the target, and 386 stars in the offset field. The mode of the proper motion distribution turned

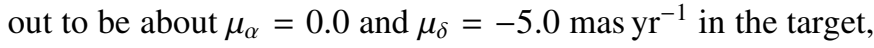



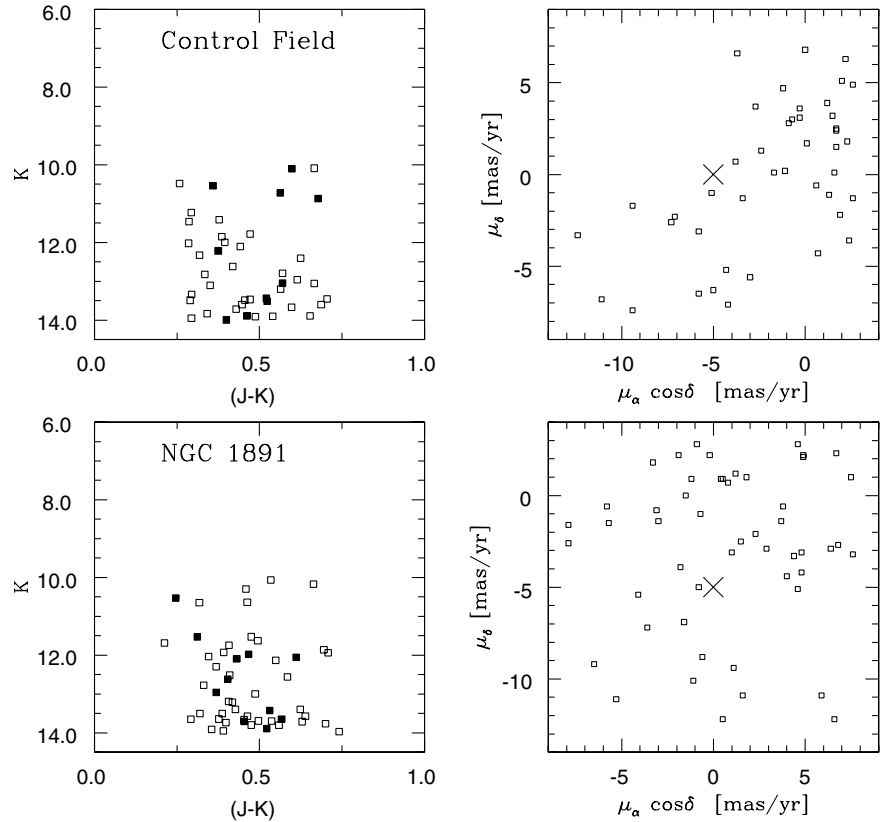

Fig. 7. Same of Fig. 6, but for NGC 1891.
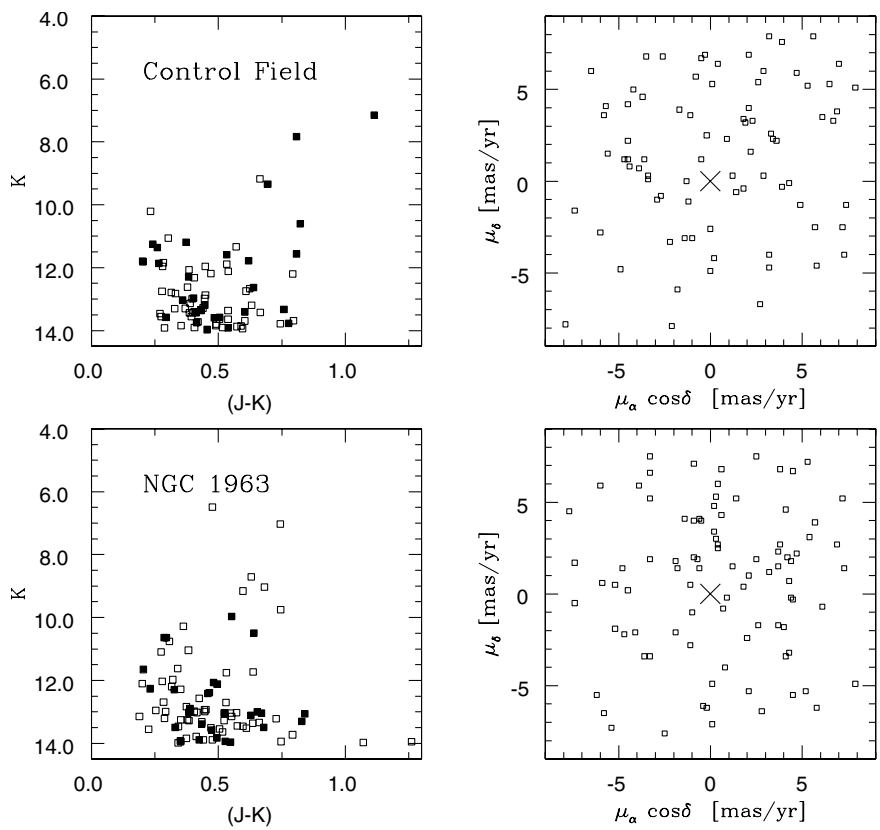

Fig. 8. Same of Fig. 6, but for NGC 1963.

and $\mu_{\alpha}=-5.0$ and $\mu_{\delta}=0.0$ mas $\mathrm{yr}^{-1}$ in the field. By inspecting Fig. 7, we see that the CMDs of target and field stars are similar, with the same spread in color and without any distinctive features. No relevant concentrations are detected in the VPDs.

We therefore propose that NGC 1891 is not a physical group.

\subsection{NGC 1963}

This target is presented in Fig. 8. We considered a $30^{\prime} \times 30^{\prime}$ area, and we found 523 stars in the target, and 437 stars in the offset field. The mode of the proper motion distribution out to

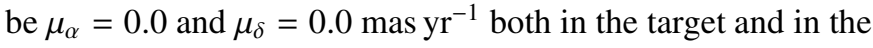
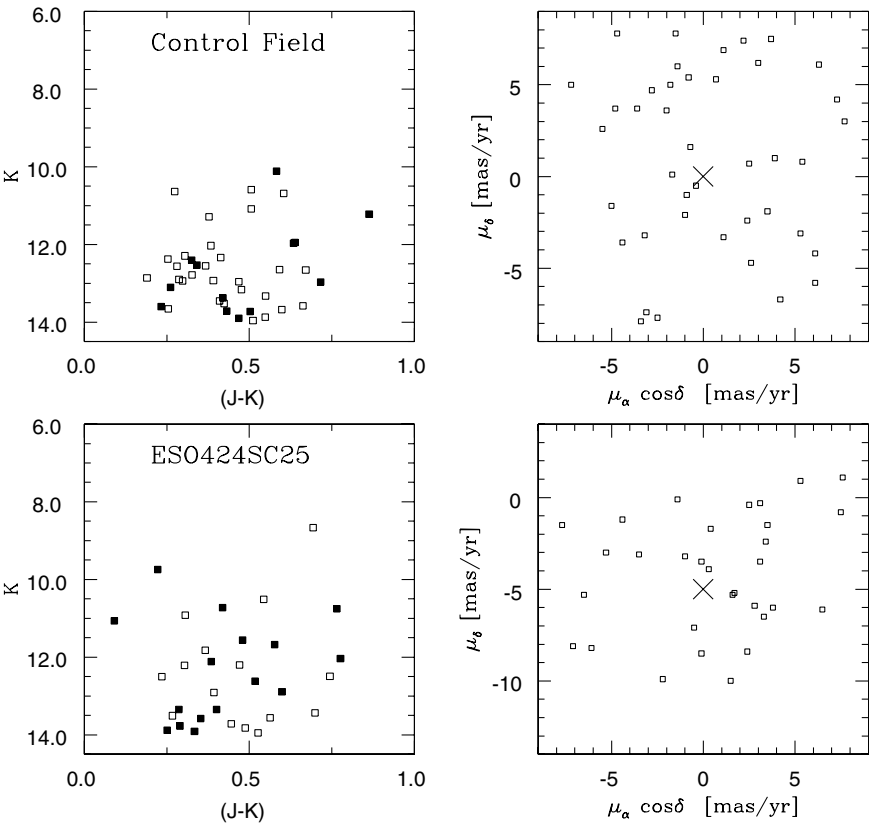

Fig. 9. Same of Fig. 6, but for ESO 424SC25.

field. By inspecting Fig. 8, we readily see that distribution of the stars both the CMDs and the VPDs are very similar, with basically the same scatter in proper motions and color.

Therefore we suggest that NGC 1963 is not a physical group.

\subsection{ESO $424 S C 25$}

This object is shown in Fig. 9. We considered a $20^{\prime} \times 20^{\prime}$ area, and we found 172 stars in the target, and 244 in the control field. The mode of the proper motion distribution turned out to be $\mu_{\alpha} \approx 0$. mas $\mathrm{yr}^{-1}$ and $\mu_{\delta} \approx-5$. mas $\mathrm{yr}^{-1}$ in the target and $\mu_{\alpha}=0.0$ and $\mu_{\delta}=0.0$ mas yr$^{-1}$ in the field. From the analysis of Fig. 9, we can conclude that ESO 424SC25 is clearly not a physical group since both the CMDs and the VPDs are very scattered and look similar.

\subsection{ESO $425 S C 06$}

This object is presented in Fig. 10. We considered a $15^{\prime} \times$ $15^{\prime}$ area, and we found 194 stars in the target area, and 198 stars in the off-set field. The mode of the proper motion distribution out to be $\mu_{\alpha} \approx 0.0$ mas yr$^{-1}$ and $\mu_{\delta} \approx 0.0$ mas yr$^{-1}$ in the target and $\mu_{\alpha}=0.0$ and $\mu_{\delta}=-5.0 \mathrm{mas} \mathrm{yr}^{-1}$ in the field. From the analysis of Fig. 10, ESO 425SC06 exhibits the same scatter as the control field both in the CMD and in the VPD with the lack of any concentration or particular feature, and therefore we suggest it is not a physical group.

\subsection{ESO $425 S C 15$}

This target is compared in Bica et al. (2001) with NGC 3680, a very well known open cluster (Anthony-Twarog \& Twarog 2003) which exhibits a significant depletion of low mass stars in the MS. Actually the two targets look very similar on the 

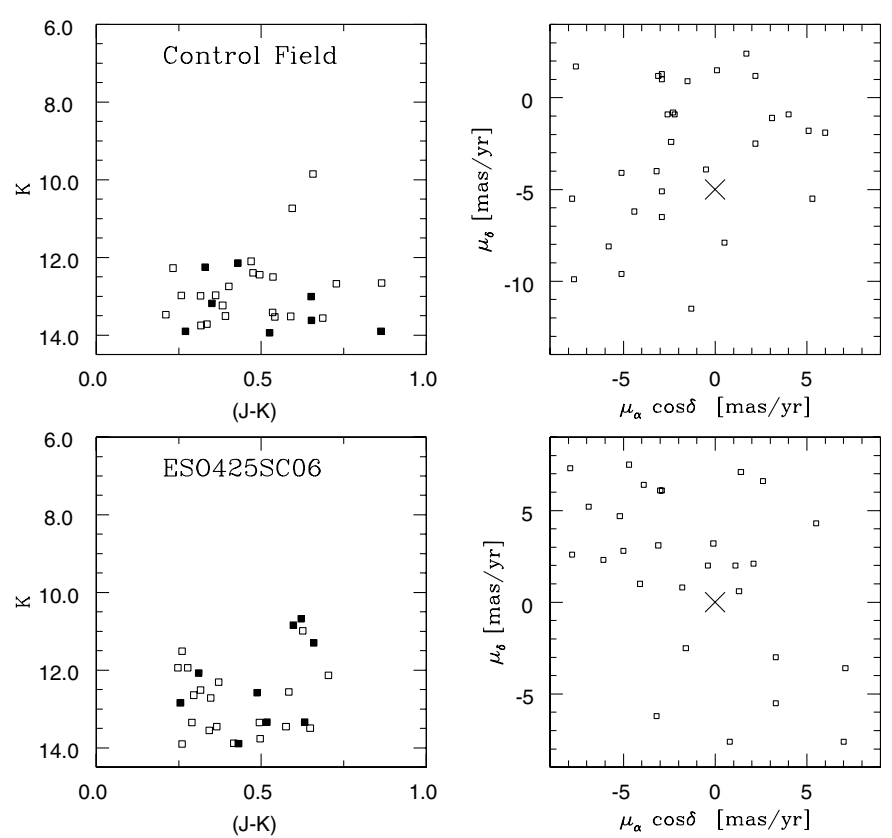

Fig. 10. Same of Fig. 6, but for ESO 425SC06.

plane of the sky (Figs. 2 and 3 in Bica et al. 2001), thus leading to the possibility that ESO $425 \mathrm{SC} 15$ might be a genuine cluster. This target is depicted in Fig. 11 . We considered a $15^{\prime} \times 15^{\prime}$ area, and we found 213 stars in the target area, and 214 stars in the nearby field. The mode of the proper motion distribution out to be $\mu_{\alpha} \approx-5.0$ mas yr$^{-1}$ and $\mu_{\delta} \approx 0.0$ mas yr$^{-1}$ in the target and $\mu_{\alpha}=0.0$ and $\mu_{\delta}=0.0 \mathrm{mas} \mathrm{yr}^{-1}$ in the field. From the analysis of CMDs and VPDs we conclude that there is not cluster in the direction of ESO 425SC15. As a confirmation of this finding, we plot in Fig. 12 the VPDs from Tycho 2 (Høg et al. 2000) of the 7 brightest stars which defines the concentration and compare it with the same number of bright stars in NGC 3680. It is readily visible that ESO $425 \mathrm{SC} 15$ is a chance alignment of different proper motion stars.

\subsection{ESO $437 S C 61$}

This target is depicted in Fig. 13. We considered a $15^{\prime} \times 15^{\prime}$ area, and found 133 stars in the target, and 160 in the control field. The mode of the proper motion distribution turned out to be $\mu_{\alpha} \approx-5.0$ mas yr$^{-1}$ and $\mu_{\delta} \approx 0.0$ mas yr$^{-1}$ in the target and

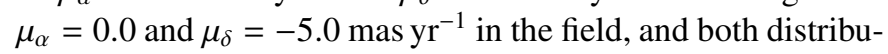
tions are very scattered. As a result, and from the appearance of CMDs and VPDs we suggest that this group is not a physical system.

\subsection{ESO $442 S C 04$}

This target is discussed in Fig. 14. We considered a $20^{\prime} \times 20^{\prime}$ area, and we found 283 stars in the target, and 226 stars in the nearby field. The mode of the proper motion distribution turned out out to be $\mu_{\alpha} \approx-5.0$ mas yr$^{-1}$ and $\mu_{\delta} \approx-5.0$ mas yr$^{-1}$ in the target and $\mu_{\alpha}=-5.0$ and $\mu_{\delta}=-10.0 \mathrm{mas} \mathrm{yr}^{-1}$ in the field. Also in this case, we cannot see any particular feature in the VPDs
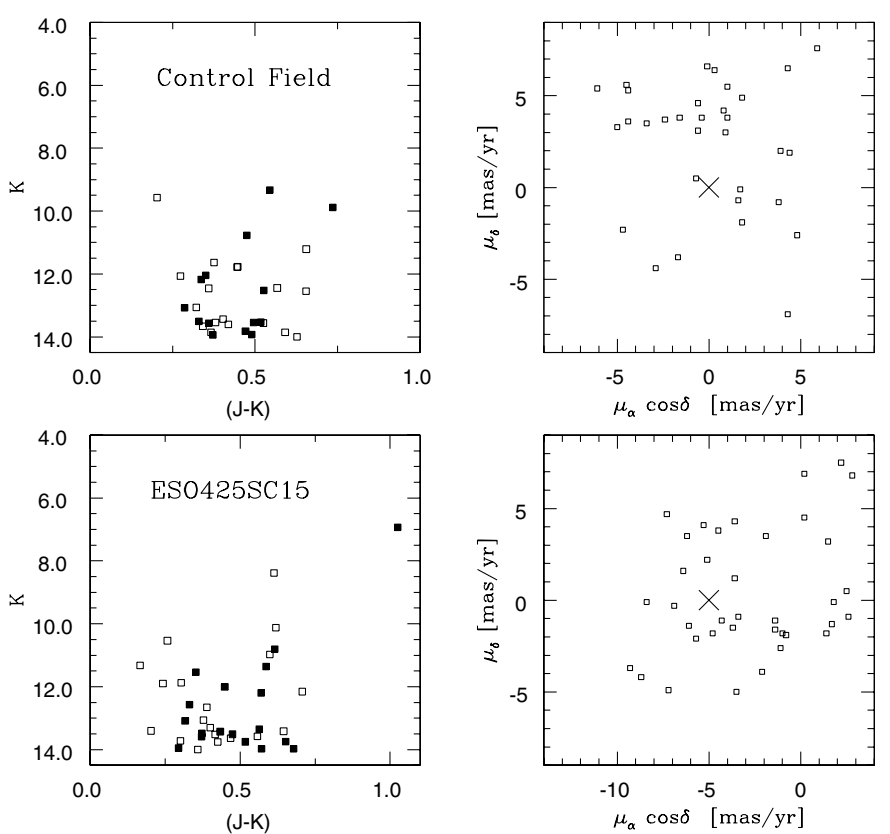

Fig. 11. Same of Fig. 6, but for ESO 425SC15.

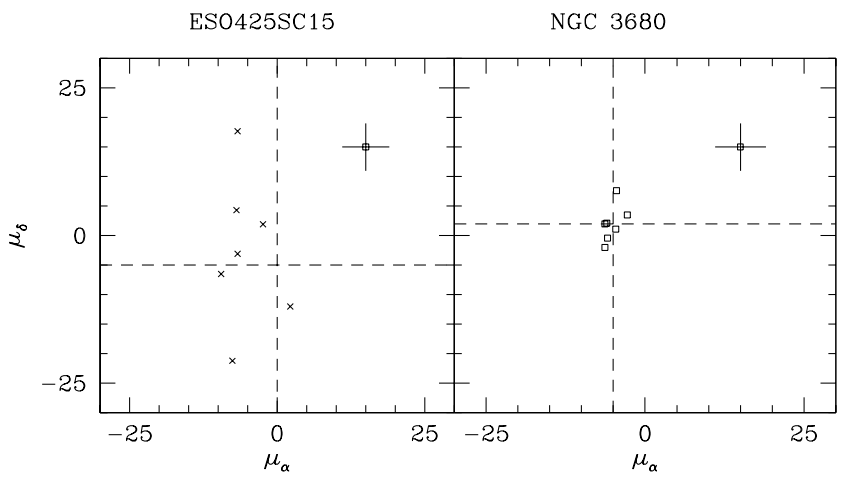

Fig. 12. VPD for ESO $425 \mathrm{SC} 15$ and NGC 3680 from Tycho 2. The cross indicate the $2 \sigma$ error bars. Dashed lines represent the mean proper motions of the two targets.

and CMDs, which do not look different, and therefore suggest that ESO 442SC04 is not a physical system.

\subsection{IC 1023}

This target is shown in Fig. 15 . We considered a $15^{\prime} \times 15^{\prime}$ area, and we found 432 stars in the target area, and 415 stars in the off-set field, roughly the same number of stars. The mode of the proper motion distribution turned out to be about

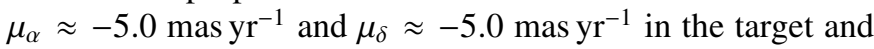
$\mu_{\alpha}=-5.0$ and $\mu_{\delta}=-5.0$ mas yr$^{-1}$ in the field. By inspecting Fig. 15, we see that the stars are scattered both in the field and in the target. No distinctive features are present in the CMDs, and we must conclude also in this case that we are not facing any physical group. 

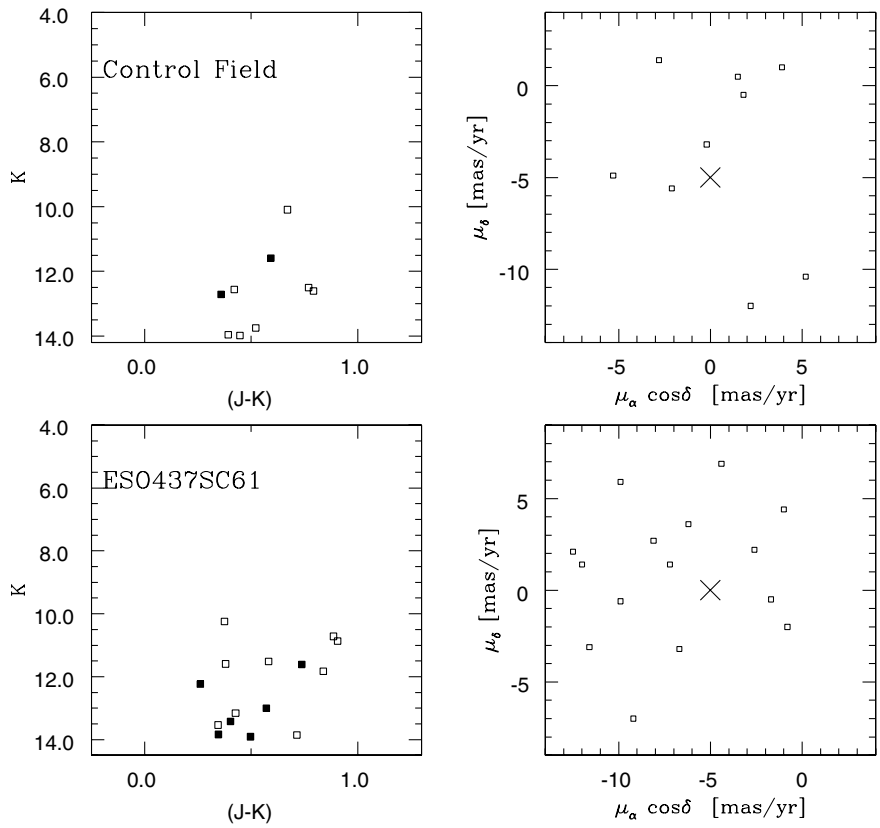

Fig. 13. Same of Fig. 6, but for ESO 437SC61.
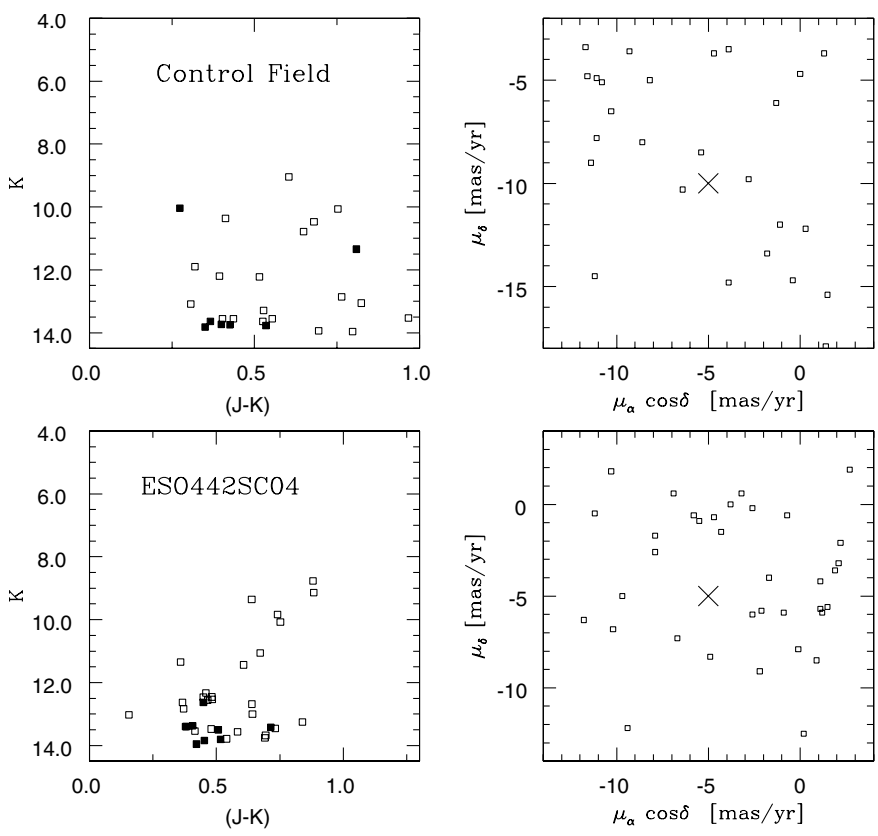

Fig. 14. Same of Fig. 6, but for ESO 442SC04.

\subsection{ESO $282 S C 26$}

This target is discussed in Fig. 16. We considered a $30^{\prime} \times$ $30^{\prime}$ area, and we found 2008 stars in the target area, and 2095 stars in the off-set field, roughly the same number of stars. The mode of the proper motion distribution turned out to be

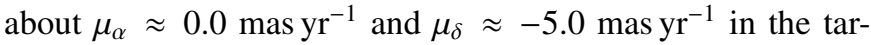
get and $\mu_{\alpha}=-5.0$ and $\mu_{\delta}=0.0$ mas yr$^{-1}$ in the field. Interestingly, in the VPDs we can see that the target is more populated than the field, and therefore this is a very promising object to be considered. At odds with all the previous cases, the CMD is very interesting. The CMD of the target and the field are at first glance very similar, although the target possesses a
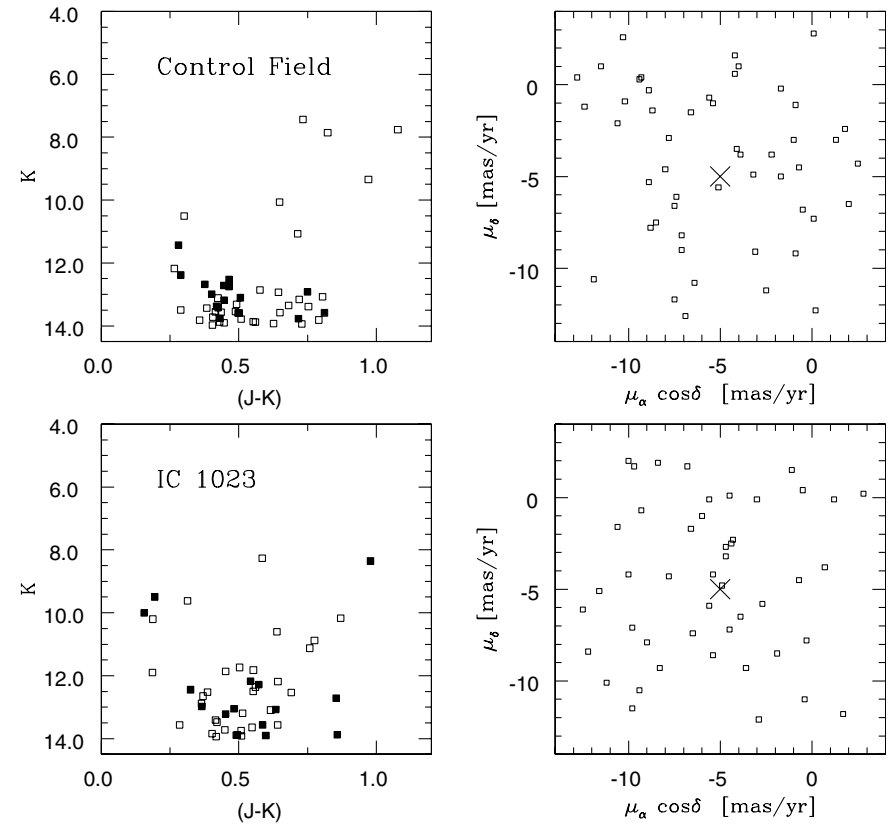

Fig. 15. Same of Fig. 6, but for IC1023.
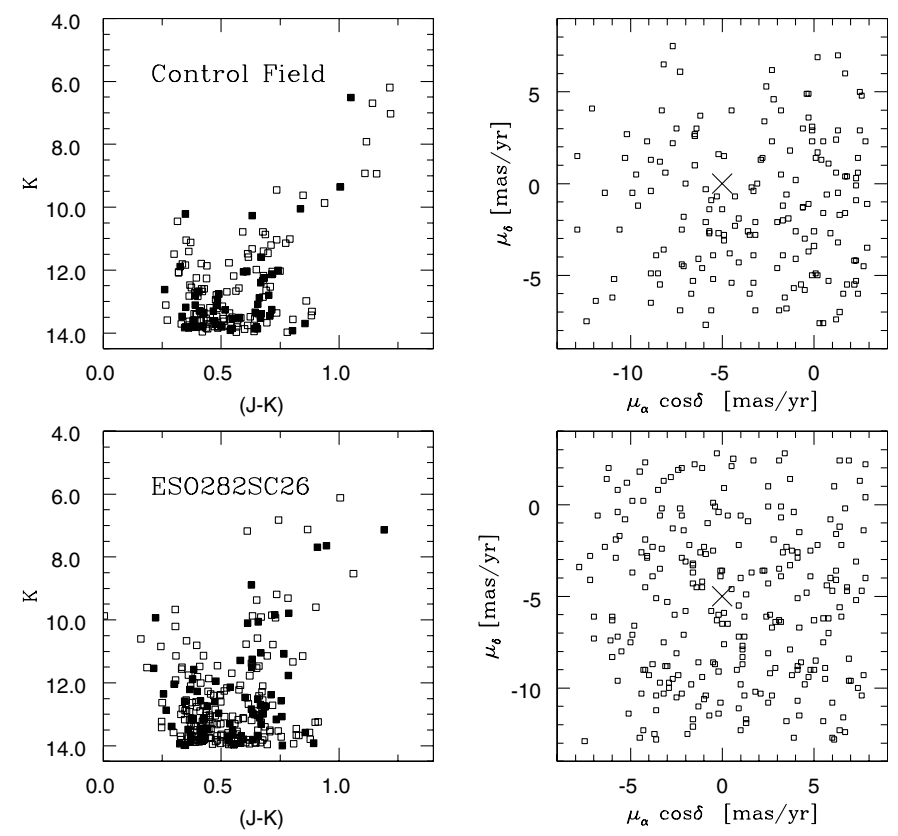

Fig. 16. Same of Fig. 6, but for ESO 282SC26.

more populated blue sequence than the field. The red part of the CMD is somewhat more different, since the cluster have more red stars within 1 SPM3 $\sigma$, and the distribution of these stars closely resembles a Red Giant Branch feature.

We therefore decided to try an isochrone fit through the stars having proper motion within 1 SPM $3 \sigma$ from the mode of the distributions and located within 7.5 arcmin from the object nominal center to limit the effect of field star contamination.

This is shown in Fig. 17, where the isochrone is for solar abundance and for an age of $1.3 \mathrm{Gyr}$, and has been taken from Bonatto et al. (2004).

The fit is very good, although some scatter is still present. As a by-product, we find that the cluster is $1.4 \mathrm{kpc}$ away from 


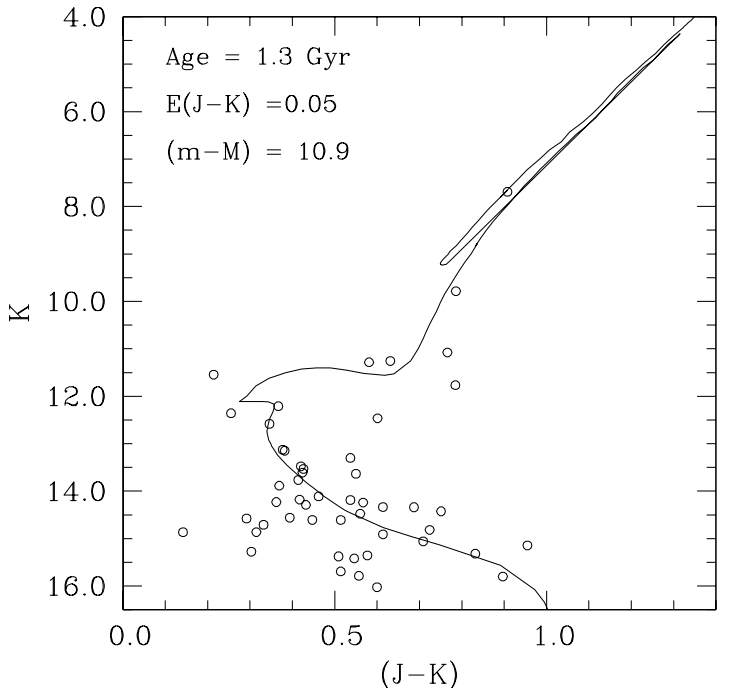

Fig. 17. 2MASS CMD of common proper motion stars in the field of ESO 282SC26 within 7.5 arcmin from the cluster nominal center.

the Sun, and it is located inside the solar ring. Therefore we suggest that ESO 282SC26 is a probable open cluster which deserves further investigation.

\subsection{ESO 464SCO9}

This target is depicted in Fig. 18. We considered a $15^{\prime} \times 15^{\prime}$ area, and we found 150 stars in the target area, and 148 stars in the off-set field, i.e. the target and the field are equally populated. The mode of the proper motion distribution turned out to be

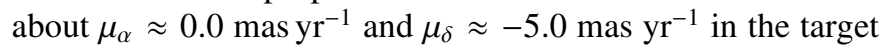
and $\mu_{\alpha}=-5.0$ and $\mu_{\delta}=0.0$ mas yr$^{-1}$ in the field, in both cases with a very large scatter. This object is very poorly populated, and we suggest we are facing a random enhancement of field stars.

\section{Conclusions}

In this paper we made use of the SPM3 catalog to assess the nature of eleven POCRs extracted from Bica et al. (2001) investigation. We first demonstrated the capability of the catalog to unravel a physical group by studying the open cluster Blanco 1. Then we analyzed VPDs and CMDs of stars selected on a common proper motion basis.

However, within the SPM typical uncertainty, a close scrutiny of the VPDs and CMDs clarifies that only one ESO 282SC26 - out of eleven candidates looks like a physical group, and for it we provide estimates of its fundamental parameters.

Acknowledgements. The authors thanks the anonymous referee for very useful suggestions which contributed to significantly improve the paper presentation. The work of GC is supported by Fundación Andes. This study made use of Simbad and WEBDA.
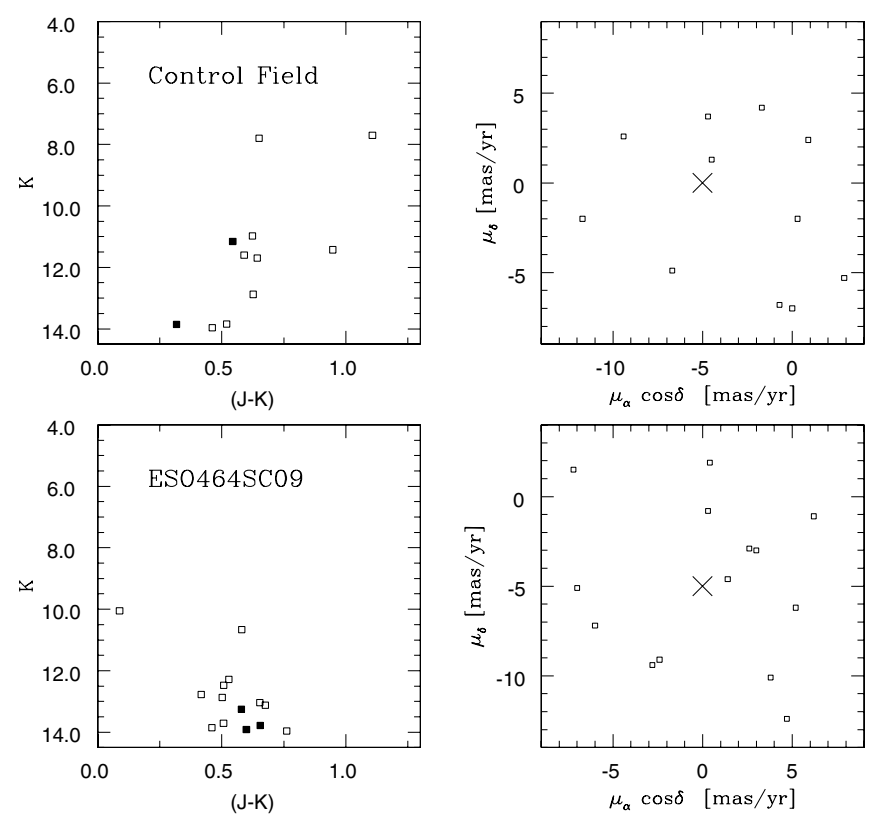

Fig. 18. Same of Fig. 6, but for ESO 464SC09.

\section{References}

Anthony-Twarog, B. J., \& Twarog, B. A. 2004, AJ, 127, 1000

Baumgardt, H. 1998, A\&A, 340, 402

Baumgardt, H., Dettbarn, C., \& Wielen, R. 2000, A\&AS, 146, 251

Bonatto, C., Bica, E., \& Girardi, L. 2004, A\&A, 415, 571

Bica, E., Santiago, B. X., Dutra, C. M., et al. 2001, A\&A, 366, 827

Carraro, G. 2000, A\&A, 357, 145

Dias, W. S., Lepine, J. R. D., \& Alessi, B. S. 2001, A\&A, 376, 441

Dias, W. S., Lepine, J. R. D., \& Alessi, B. S. 2002a, A\&A, 386, 168

Dias, W. S., Alessi, B. S., Moitinho, A., \& Lepine, J. R. D. 2002b, A\&A, 389, 871

Girard, M. T., Dinescu, I. D., van Altena, W. F., et al. 2004, AJ, 127, 3060

Høg, E., Fabricius, C., Makarov, V. V., et al. 2000, A\&A, 355, L27

van Leeuwen, F. 1999, A\&A, 341, L71

Odenkirchen, M., \& Soubiran, C. 2002, A\&A, 383, 163

Pavani, D. B., Bica, E., Dutra, C. M., et al. 2001, A\&A, 374, 554

Platais, I., Kozhurina-Platais, V., \& van Leeuwen, F. 1998, AJ, 116, 2423

Schmidt-Kaler, Th. 1982, Landolt-Börnstein, Numerical data and Functional Relationships in Science and Technology, New Series, Group VI, Vol. 2b, ed. K. Schaifers, \& H. H. Voigt (Berlin: Springer Verlag), 14

Skrutskie, M., Schneider, S. E., Stiening, R., et al. 1997, in The Impact of Large Scale Near-IR Sky Surveys, ed. Garzon et al. (Netherlands: Kluwer), 210, 187

Villanova, S. 2003, Master Thesis, Padova University

Villanova, S., Carraro, G., de la Fuente Marcos, R., \& Stagni, R. 2004a, A\&A, 428, 67

Villanova, S., Carraro, G., \& de la Fuente Marcos, R. 2004b, in Milky Way Surveys: Structure and Evolution of our Galaxy, ed. D. Clemens, R. Y. Shah, \& T. Brainerd (San Francisco: Astronomical Society of the Pacific), ASP Conf. Proc., 317, 196 